American Journal of Pediatrics
2020; 6(4): $408-420$
http://www.sciencepublishinggroup.com/j/ajp
doi: 10.11648 /j.ajp.20200604.14
ISSN: $2472-0887$ (Print); ISSN: $2472-0909$ (Online)

\title{
The Impacts of Home Confinement Due to Coronavirus (COVID-19) on Children: A Cross Sectional Survey Study, Mediclinic City Hospital, Dubai, UAE
}

\author{
Sam Hassan*, Mary Saviour, Sanjay Perkar, Tessy Augustine, Samie Ullah Ahmed, \\ Bindu Radhadevi, Shaheena Batool, Rawan Abunqira, Erica Delos Santos, Helen Strike, \\ Manal Blelepsma
}

Mediclinic City Hospital, Dubai, UAE

\section{Email address:}

Sam.hassan@mediclinic.ae (S. Hassan), mary.saviour@mediclinic.ae (M. Saviour), sanjay.perkar@ mediclinic.ae (S. Perkar), tessy.augustine@mediclinic.ae (T. Augustine), samie.ahmed@mediclinic.ae (S. U. Ahmed), bindu.radhadevi@mediclinic.ae (B. Radhadevi), shaheena.batool@mediclinic.ae (S. Batool), rawan.abunqira@mediclinic.ae (R. Abunqira), erica.santos@mediclinic.ae (E. D. Santos), helen.strike@mediclinic.ae (H. Strike), manal.blelepsma@mediclinic.ae (M. Blelepsma)

${ }^{*}$ Corresponding author

\section{To cite this article:}

Sam Hassan, Mary Saviour, Sanjay Perkar, Tessy Augustine, Samie Ullah Ahmed, Bindu Radhadevi, Shaheena Batool, Rawan Abunqira, Erica Delos Santos, Helen Strike, Manal Blelepsma. The Impacts of Home Confinement Due to Coronavirus (COVID-19) on Children: A Cross Sectional Survey Study, Mediclinic City Hospital, Dubai, UAE. American Journal of Pediatrics. Vol. 6, No. 4, 2020 , pp. 408-420. doi: 10.11648/j.ajp.20200604.14

Received: September 20, 2020; Accepted: October 7, 2020; Published: October 17, 2020

\begin{abstract}
Children are developing individuals with countless factors affecting their growth and development, from genetics to parenting, schools, environments, nutrition, good habits of sleep and health. They are very sensitive individuals to sudden changes in routine. Schools represent life for them and a place of not only learning, but also social interaction and sensory neurodevelopment. Disasters and pandemics such as the existing situation of COVID-19 are a major cause of trauma affecting their education, play, mental health, physical health, vaccinations, sleep, and development. The governments' strategies particularly home confinement and school closure to prevent the COVID-19 pandemic spread added unavoidable stress and psychosocial impacts on both adults and children. Trauma of this kind may endure for a long time with negative consequences that may manifest later on, even up to adult life. We conducted a cross sectional parental survey, to assess the impacts of home confinement on children in the cosmopolitan city of Dubai, UAE. Children included were from 3 years until 16 years old who were in schools or pre-school placements before COVID-19 started. Total number of children included in the survey was 658 of which 327 were boys and 331 girls. We found that the impact of the home confinement on children was significant and directly affected their quality of life (QoL) that may extend beyond the lockdown for longtime. This study will help relevant authorities and organizations to understand the negative impacts brought by the COVID-19 confinement on children and to adopt appropriate strategies to help children and their parents tackle these impacts and get them back to normal life and school again. This study also paved the way for future studies in the identification and management of children's behavior, attention, education, and other factors that play active roles in QoL and normal development. Moreover, this study may help in embracing early preventative and management plans by schools and authorities in future similar pandemics, infections, disasters or school outbreaks. We also discuss strategies for school reopening and flexibility when an outbreak happens again in a school or community.
\end{abstract}

Keywords: Coronavirus, COVID-19, SARS-CoV-2, Confinement, Lockdown, Children, Vaccinations, Schools, Quality of Life 


\section{Introduction}

Coronavirus disease 2019 (COVID-19) is an emerging infectious disease caused by newly discovered Severe Acute Respiratory Syndrome Coronavirus 2 (SARS-CoV-2).

It was first identified in December 2019 in Wuhan, China, then spread globally by phylogenetically mutated strains of the same virus affecting more than 35 million people, with deaths exceeding 1 million and still rapidly increasing as of October 2020 [1]. From January 2020, the World Health Organization (WHO) and affected countries implemented primary measures of social distancing, self-isolation, and nationwide lockdowns. Governments around the world ordered school closures as an emergency measure to prevent spreading of the virus. Public activities including children's centers and nurseries closed too. One and a half billion children and adolescents worldwide were confined to their homes following school closure. Most countries considered online home schooling, including the United Arab Emirates (UAE), to ensure continuity of education. The option of home online schooling continued to the $\mathrm{n}$ discovered Severe Acute Respiratory Syndrome Coronavirus 2 (SARS-CoV-2).

The first reported mortality was on 20 March 2020, and lockdown started on 22 March 2020. School closures were announced on 4 March 2020 and remained closed until September 2020 despite lockdown measures being eased in late June 2020, where children under 12 years allowed for the first time to go to Malls and shopping centers. The online home schooling is an optional choice for the current academic term and may be extended to the whole academic year 2020/2021.

Colossal efforts were made by schools and teachers at all levels to create online courses to help to alleviate many parents' concerns about their children's educational achievement by certifying that school education is principally uninterrupted. However, prolonged school closure and home confinement for many months may result in negative impacts on children's physical, behavioral and mental health.

Previous pandemic disasters and subsequent diseasecontainment responses led to conditions that families and children find traumatic to the health needs of children and their families. During the COVID-19 pandemic the most important effect of lockdown was school closure. Previous studies suggested that when children are out of school during weekends and summer holidays they become physically less active, have much longer time on iPads and screen games, might get uneven sleep patterns, and unhealthy picking diets that resulted in weight gain and a loss of fitness [2]. These harmful consequences are likely to be even worse when children are confined to their homes without access to outdoor activities and without interaction with same age groups during a pandemic. The online schooling, though offers continuity of the basic education but will never be a replacement for sensory engaged educational achievement and neuro-development.

The lockdown, school closure and the pandemic all resulted in psychological, behavioral and physical trauma that may be easily overlooked. Psychological stress may result from fear of infection, anger and boredom, incompetent information, lack of face-to-face contact with classmates, friends, teachers and relatives and inadequate personal space at home. In addition, possible family financial loss which is very problematic and has long-term effects on children and their families. Previous studies [3] showed that the mean post-traumatic stress scores were four times higher in children who were quarantined than in those who were not quarantined. Confinement has instability and uncertainty that may cause discomfort, anger, depression, inattention, behavioral problems, boredom, emotional liability, fears, eating problems and sleeping disorders. Studies in adults showed widespread symptoms such as anxiety, frustration, panic attacks, loss or increase of appetite, sleep disorders, depression, loneliness, delusions, fear, suicidal thoughts, and domestic violence as a result from home confinement [4-7]. Similar studies in children are scarcely but essentially to be done to help understand impacts of lockdown to develop proper preventative actions [8].

The aim of our study is to assess the impacts resulting from this unprecedented lockdown on children which are important for professionals such as schools, health services, parents and organizations. As far as our search in English literature is concerned, this is the first study about parents' perspective in a multicultural city of Dubai. Though vulnerable group for impact, young children have little voice to advocate their needs. Parents are the best people to describe the impact of the lockdown on their children particularly as they become closer to them during the lockdown and in so many instances acted as their teacher. In this survey we studied the impacts of home confinement from the parent's perspectives.

\section{Methodology}

This is a cross sectional descriptive research study, implemented by parental questionnaire survey, addressing the impacts of the home confinement due to COVID-19 pandemic on children of school age from 3 to 16 years from a randomly drawn stratified sample. In designing the questionnaire, we took into consideration multicultural differences and lack of unified standard criteria to assess children's QoL from parents' perspectives. We reviewed relevant published literature, and designed a questionnaire based on generic criteria to assess how children responded to the confinement. These criteria (figure 1) were based on the standard daily activities, requirements, behaviors, sleep, eating habits, education, mental and psychosomatic factors and were modified according to the Diagnostic and Statistical Manual of Mental Disorders (DSM-5) criteria commonly used for a cross-cultural assessment of mental and psychological disorders [9]. Reliability and feasibility of the modified newly adopted questionnaires were tested by a pilot study of 20 parents and modified again to enhance clarity.

The questionnaire was anonymous to avoid any bias, influence or hesitancy and made as simple as possible, 
addressing imperative sociodemographic data and important areas in children's daily life, behavior, activities, learning, sleep, eating and social aspects. The questions were framed in a binary choice (yes/no) and choice question of several options. Parents surveyed were multinational and nearly all of them English speaking however if needed translation was available. If both parents were present we took the answer from both and nearly always there was parental agreement. Explanation about the survey and verbal consent was taken to fill the questionnaires. Parents had the option to fill the survey by themselves or by short interview with a doctor or a nurse.

The study was carried out by Mediclinic City Hospital in Dubai, United Arab Emirates from June until September 2020. Of the total 700 participants, 32 did not return the filled questionnaires, and 10 could not be included in the study as the submitted forms were incomplete. Thus, a total of 658 participants were finally included in the study. The sample size is sufficiently large enough to estimate the prevalence of the conditions of interest with adequate precision. It was calculated taking inconsideration that the estimated Dubai 2018 child population up to 16 years old is just above 600,000 with a population of 2.8 million [10], for a $95 \%$ confidence level at confidence interval of 5 . For the purpose of this study, a sample of 600 was calculated for an unbiased representation that can be easily observed and measured. Children with chronic illnesses and disabilities were excluded as this study about children in mainstream schools without co-morbidities.

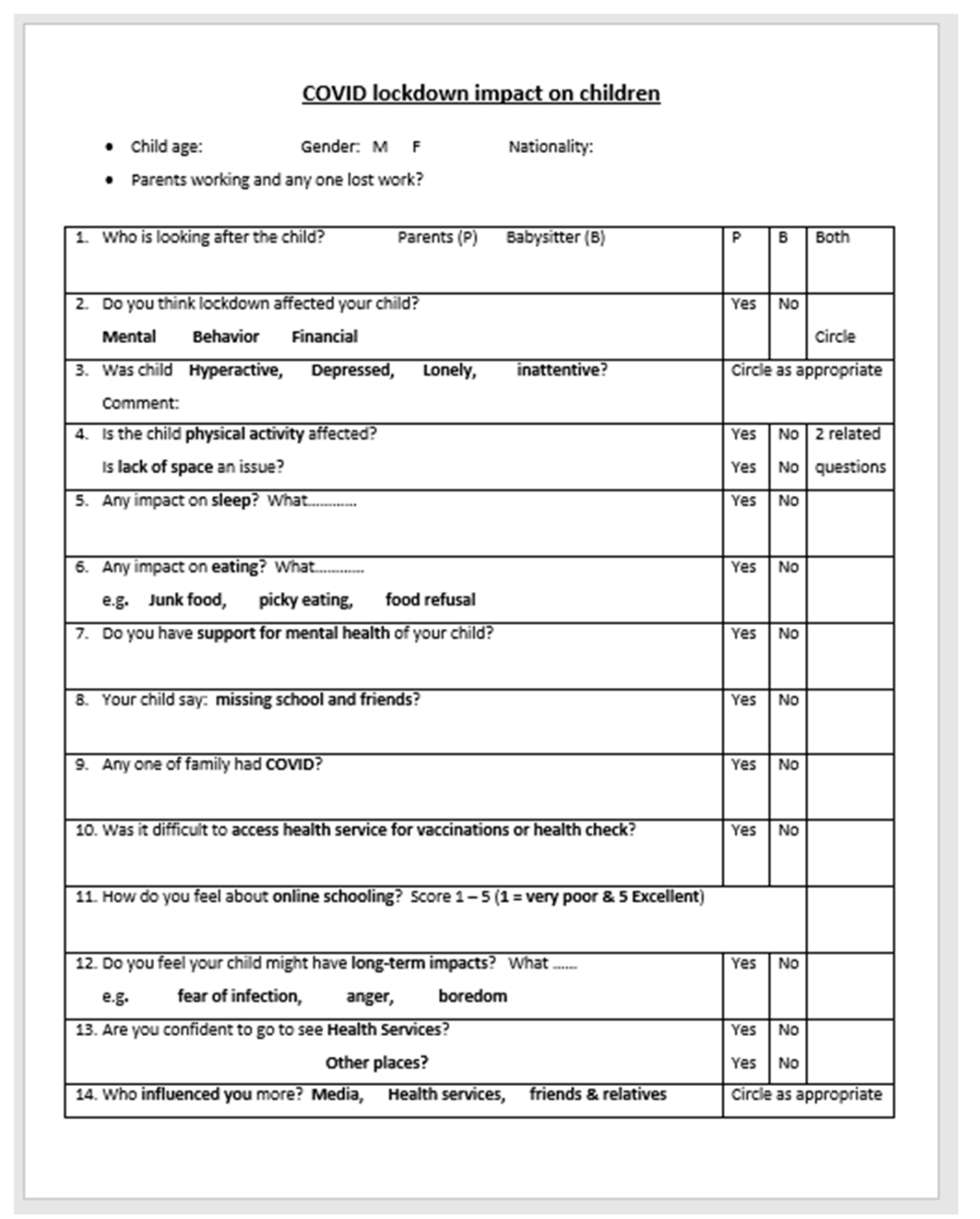

Figure 1. Survey questioner template to assess COVID-19 home confinement impacts on children.

During the informed verbal consent process, survey participants were assured all data would be used only for research purposes and answers remained anonymous and confidential. The study is a survey questionnaire and no 
names, contact information, personal information or medical data are required. The survey forms and the data sheets were kept strictly confidential and only accessible to the assigned principle investigators and Research Coordinator. The declaration of Helsinki and Good Clinical Practice guidelines were followed. Descriptive statistics were used to define the proportion of responses for each question and the total distribution of the total score of each questionnaire.

\section{Statistical Analysis}

A sample size of 658 was determined based on the findings of the pilot study conducted by us on 50 children in Dubai, which revealed that the prevalence of impacts of COVID-19 lockdown on the well-being of children in the community was $50 \%$. We applied the Cochran formula (Michener, 1976) for the calculation, using the above $50 \%$ prevalence rate, a precision value of 0.05 and a Z-value of 1.96 , with level of confidence or risk and degree of variability as $95 \%$ as encompassed under the Central Limit Theorem.

Survey data was analyzed using SPSS- version 27, a statistical analysis software. The data was grouped thematically and coded. The dependent variable was dichotomized. Binary logistic regression analysis was performed using all relevant socioeconomic variables and significant variables with $p$ value $(p<0.001)$ were considered in the logistic regression analysis. The result of the analysis was then used to identify the different possible risk factors related with Covid-19 lock down. The influence of the risk factors was described in terms of the odds ratio and adjusted odds ratio along with confidence intervals.

The sociodemographic characteristics including the indicators of age, gender, nationality, parent employment status, child care, influence of media and proxy measures of economic status of the participants were summarized in the (Table 1 and Table 2).

The study demonstrated the Prevalence of COVID-19 affected children in Dubai was $72 \%$, in contrast to the pilot study which showed $50 \%$, which revealed several implications on psychological and physiological distress among the children community. This addresses determinants such as mental, behavior, hyperactivity, depression, inattentiveness and sleep etc A comprehensive statistical analysis was performed using a logistic regression model and the following inferences were recorded (Table 3). Our study categorically disclosed that child behavior emerged to be the strongest risk factor in the impact of COVID-19 Lockdown, (refined OR 69.5, 31.3 to 75.1), while depression was identified as the second strongest risk factor associated with newly emerging symptoms in children (table 3 ). The other identified characteristics and associations of significance were inattentiveness, loneliness, hyperactive and mental status respectively. The significant association of increased risk of child mental depression (refined - OR 4.9 in the interval 2.9 to 8.2 ) disappeared with other confounding newly emerging symptoms.

A novel observation in our study was that children who were reported to be affected mentally had significantly greater odds ratios of having association with COVID-19 lock down, but very few pursued sought support for mental health due to difficulty or/and lack of confidence to access health support. Table 3 shows that an appreciable statistical significance on "missing school and friends" while the student community encountered an extraordinary mental and physiological lock down and craved for social interaction.

The original interpretation as shown in table 3, defined that the independent variables and their association with the impact of COVID-19 lockdown affected, the dependent variable with $\mathrm{P}$-value $(\mathrm{p}<0.001)$ statistically significant.

Table 1. Patients per nationalities.

\begin{tabular}{llll}
\hline Algeria & 1 & Nepal & 2 \\
Argentina & 1 & Netherland & 3 \\
Australia & 5 & New Zealand & 2 \\
Bahrain & 2 & Nigeria & 4 \\
Bangladesh & 1 & Oman & 2 \\
Belarus & 1 & Pakistan & 82 \\
Brazil & 2 & Palestine & 3 \\
Canada & 6 & Portugal & 2 \\
Denmark & 3 & Romania & 2 \\
Dominican & 1 & Russia & 3 \\
Egypt & 23 & Saudi & 1 \\
Fiji & 2 & Singapore & 3 \\
Filipino & 68 & Siri Lanka & 4 \\
French & 10 & South Africa & 5 \\
Germany & 5 & Spain & 2 \\
India & 176 & Sudan & 9 \\
Ireland & 10 & Syria & 6 \\
Italy & 2 & Tunisia & 5 \\
Japan & 1 & UAE & 84 \\
Jordan & 29 & UK & 45 \\
Kenya & 3 & USA & 14 \\
Lebanon & 8 & Uzbekistan & 1 \\
Libya & 1 & Venezuela & 2 \\
Malaysia & 10 & Total & 658 \\
Mozambique & 1 & & \\
\hline & & &
\end{tabular}

Table 2. Sociodemographic characteristics of Participants.

\begin{tabular}{llll}
\hline Variable & Female N (\%) & Male N (\%) & Total N (\%) \\
\hline Total & $331(54.3)$ & $327(49.70)$ & 658 \\
Age (years) & & & \\
Age group 3-5 & $97(29.3)$ & $78(23.9)$ & $175(26.6)$ \\
Age group 6-12 & $182(55.0)$ & $194(59.3)$ & $376(57.1)$ \\
Age group 13-16 & $52(15.7))$ & $55(16.8)$ & $107(16.3)$ \\
Nationality & & & \\
UAE & $36(10.9)$ & $48(14.6)$ & $84(12.8)$ \\
India & $100(30)$ & $76(23.2)$ & $176(26.7)$ \\
Pakistan & $38(11.4)$ & $44(13.5)$ & $82(12.6)$ \\
Philippines & $30(9)$ & $38(11.6)$ & $68(10.4)$ \\
UK & $23(6.9)$ & $22(6.7))$ & $45(6.8)$ \\
Others & $104(31.6)$ & $99(30.2$ & $203(30.8)$ \\
Parents lost job & $19(5.7)$ & $27(8.2)$ & $46(7)$ \\
Child care & & & \\
Parents & $215(64.9)$ & $200(61.6)$ & $415(63)$ \\
Baby sitter & $20(6)$ & $19(5.8)$ & $39(6)$ \\
Both & $96(29)$ & $108(32.6)$ & $204(31)$ \\
External influence & & & \\
Media & $200(60.4)$ & $146(44.6)$ & $346(40)$ \\
Health service & $151(45.6)$ & $129(39.4)$ & $280(32)$ \\
Friends and relatives & $221(66.7)$ & $226(69.1)$ & $247(28)$ \\
Online schooling & & & \\
Score below average & $61(18.4)$ & $62(19)$ & $123(18,7)$ \\
Score above average & $270(81.6)$ & $265(81)$ & $535(81.3)$ \\
\hline
\end{tabular}


Table 3. Statistical Analysis.

\begin{tabular}{|c|c|c|c|c|c|c|c|}
\hline Descriptive variable & $\begin{array}{l}\text { COVID not } \\
\text { affected N (\%) }\end{array}$ & $\begin{array}{l}\text { COVID affected } \\
\text { N }(\%)\end{array}$ & p Value & $\begin{array}{l}\text { Crude }{ }^{++} \\
\text {OR }\end{array}$ & $95 \%+C I$ & $\begin{array}{l}\text { Refined }^{++} \\
\text {OR }\end{array}$ & $95 \%+C I$ \\
\hline Total & $179(27.20)$ & $479(72.80)$ & & & & & \\
\hline Gender & $95(29.1)$ & & & & & & \\
\hline Male & $84(25.4)$ & $232(70.0)$ & 0.247 & 0.8 & 0.6 to 1.2 & 1.3 & 0.9 to 1.2 \\
\hline Female & 331 & $247(74.6)$ & & 1.2 & 1.1 to 1.8 & & \\
\hline \multicolumn{8}{|l|}{ Age (years) } \\
\hline Age group 3-5 & $58(32.95)$ & $118(67.04)$ & & 1.0 & 1.2 to 4.8 & & \\
\hline Age group 6-12 & $91(24.20)$ & $285(75.79)$ & & $2.7^{*}$ & 1.5 to 2,7 & & \\
\hline Age group 13-16 & $30(28.03)$ & $77(71.96)$ & & 1.3 & & & \\
\hline Psychological & & & $<0.001^{*}$ & & & & \\
\hline Mental & $33(12)$ & $241(88)$ & 0.224 & 4.5 & 2.3 to 6.8 & 4.9 & 2.9 to 8.2 \\
\hline Support for mental health & $25(22.5)$ & $86(77.5)$ & $<0.001$ & 1.4 & 0.8 to 2.2 & $1.8^{*}$ & 2.9 to 8.2 \\
\hline Behavior & $7(2)$ & $347(98)$ & $<0.001$ & 64.60 & 29.6 to $14^{*}$ & $69.5^{*}$ & 31.3 to $75.1^{*}$ \\
\hline $\begin{array}{l}\text { Missing school and friends } \\
\text { Newly emerging symptoms }\end{array}$ & $118(23.9)$ & $376(76.1)$ & & $1.9^{*}$ & 1.3 to $2.8^{*}$ & $1.2^{*}$ & 0.7 to 2.0 \\
\hline Hyperactive & $36(15.2)$ & $44(5.5)$ & $<0.001 *$ & 2.9 & 1.9 to 4.3 & $4.7 *$ & 2.9 to 7.4 \\
\hline Depressed & $4(3.4)$ & $115(96.6)$ & $<0.001$ & 13.8 & 5.0 to 38.1 & $15.6^{*} 11.5$ & 0.9 to 3 \\
\hline Lonely & $13(5.5)$ & $225(94.5)$ & $<0.001$ & 11.311 .4 & 5.0 to 38.1 & $*$ & 06.1 to 21.5 \\
\hline Inattentive & $5(4.1)$ & $118(95.9)$ & $<0.001$ & & 4.6 to 28.3 & $12.7^{*}$ & 4.9 to 32.9 \\
\hline \multicolumn{8}{|l|}{ Physiological } \\
\hline Physical activities & $102(21.7)$ & $369(78.3)$ & $<0.001 *$ & 2.5 & 1.8 to 3.6 & $2.2 *$ & 1.5 to 3.2 \\
\hline Lack of space & $74(22.4)$ & $256(77.6)$ & 0.006 & 1.63 & 1.2 to 2.3 & $1.8^{*}$ & 0.9 to 1.9 \\
\hline Sleep & $59(21.6)$ & $214(78.4)$ & 0.007 & 1.64 & 1.1 to 2.3 & 1.5 & 1.0 to 1.1 \\
\hline \multicolumn{8}{|l|}{ Food habits } \\
\hline Junk food & $26(15.1)$ & $146(84.9)$ & $<0.001$ & 2.6 & 1.6 to 4.08 & $2.4^{*}$ & 1.5 to 3.8 \\
\hline Picky eating & $9(10.3)$ & $78(89.7)$ & 0.002 & 3.7 & 1.8 to 7.5 & $3.1 *$ & 1.5 to 6.5 \\
\hline Food refusal & $14(17.5)$ & $66(82.5$ & 0.096 & 1.9 & 1.03 to 3.5 & 1.7 & 0.91 to 3.1 \\
\hline \multicolumn{8}{|l|}{ Environment } \\
\hline Difficulty to access health & $57(28.2)$ & $145(71.8)$ & 0.697 & 0.9 & 0.6 TO 1.3 & 0.95 & 0.7 to 1.4 \\
\hline Confidence to visit health & $121(27.1)$ & 325 (72.9) & 0.951 & 1.0 & 0.7 TO 1.4 & 0.98 & 0.7 to 1.4 \\
\hline $\begin{array}{l}\text { Confidence to visit other place } \\
\text { Long term impact }\end{array}$ & $43(25.1)$ & $128(75.9)$ & 0.482 & 1.1 & 0.8 TO 1.7 & 1.1 & 0.7 to 1.8 \\
\hline Fear of infection & $55(22.3)$ & $192(77.7)$ & 0.027 & 1.5 & .0 to 2.1 & 1.3 & 0.9 to 1.9 \\
\hline Anger & $11(10.2)$ & $97(89.8)$ & $<0.001 *$ & 3.9 & 2 to 7.4 & $3.6^{*}$ & 1,9 to 7.0 \\
\hline Boredom & $65(26)$ & $185(74)$ & 0.587 & 1.1 & 0.8 to 1.6 & 1.0 & 0.7 to 1.4 \\
\hline Presence COVID in family & $14(24.1)$ & $44(75.9)$ & 0.583 & 1.2 & 0.6 to 2.2 & & \\
\hline Finance & $0(0)$ & $66(100)$ & 0.997 & & & & \\
\hline
\end{tabular}

* Signifies evident statistical significance.

OR - Odds ratio CI- Confidence Interval.

\section{Results}

Total number of children included 658 children in a cohort cross sectional study of a simple questioner for the parents. There were 327 boys (49.7\%) and 331 girls (50.3\%), age 3-16 years old, $24.9 \%(164 / 658)$ are pre-primary school age, $60.5 \%$
(398/658) were primary school age and 14.5\% (96/658) were secondary school age (Figure 2). They were all residents or citizens in Dubai during and before the lockdown. All children were in school or pre-school settings. Parents' sociodemographic characteristics are in Tables 1 and 2.

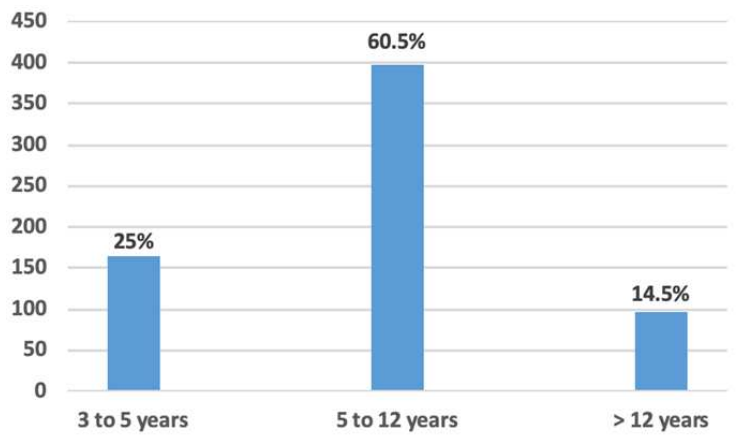

(A)

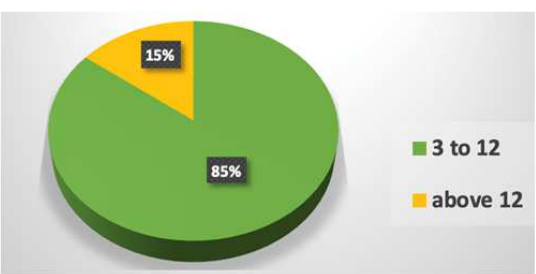

(B)

Figure 2. (A) all children, (B) $85 \%$ of children were in preprimary or primary (age in years). 
Among the 658 parents, $7 \%(46 / 658)$ of one or both parents lost employment and more than that number were on reduced pay.

\section{Behavioral, Mental and Financial Difficulties:}

More than $72 \%$ (479/658) of parents believe that the COVID19 home confinement affected their children in a negative way (figure $3 \mathrm{~A}$ ). The reported negative impacts in order of frequency are behavior; mental and financial (figure $3 \mathrm{~B}$ ).
Among those who answered yes, 74\% $(n=354 / 479)$ answered yes for behavioral difficulties which was not noted before the lockdown, 57\% ( $\mathrm{n}=274 / 479)$ of the parents noted variable degrees of mental distress while $(14 \%)(n=66 / 479)$ had financial difficulties mostly when one or both parents lost jobs or on reduced pay (figure $3 \mathrm{C}$ ). Very few parents only reported positive effects with confinement, which occurred when there is more than one child at home, socializing and playing together.

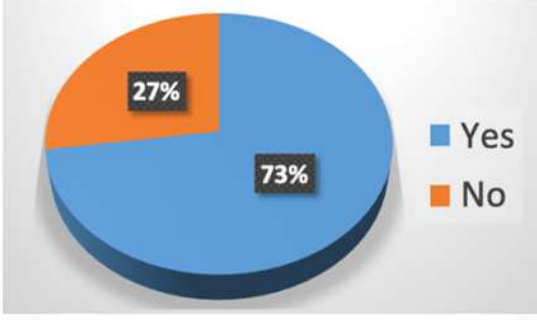

(A)

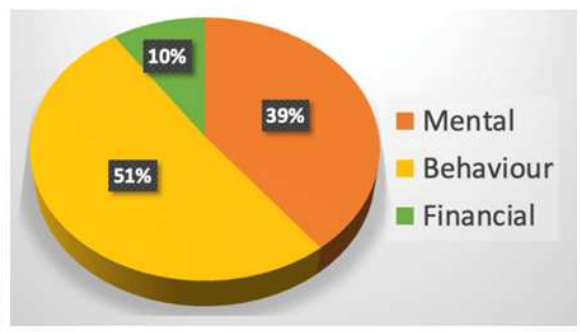

(B)

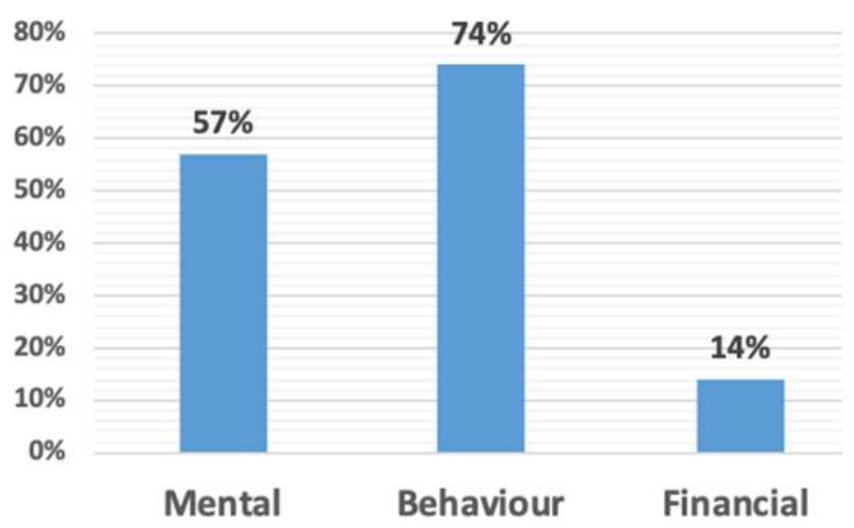

(C)

Figure 3. (A) parents answers for lockdown negative impacts. (B) Behavioral, Mental and Financial impact percentages and (C) percentages reported among parents answered yes.

\section{Hyperactivity, Inattentiveness, Depression and Lonely} Feeling:

Hyperactivity and lonely feeling were equally reported $(33 \%)(n=237 / 658)$ and $(n=238 / 658)$ respectively (figure 4$)$ while depression and inattentiveness were reported in around $(17 \%)$ each $(n=123 / 658)$ and $(n=119 / 658)$ respectively. There was insignificant difference between boys and girls or culturally.

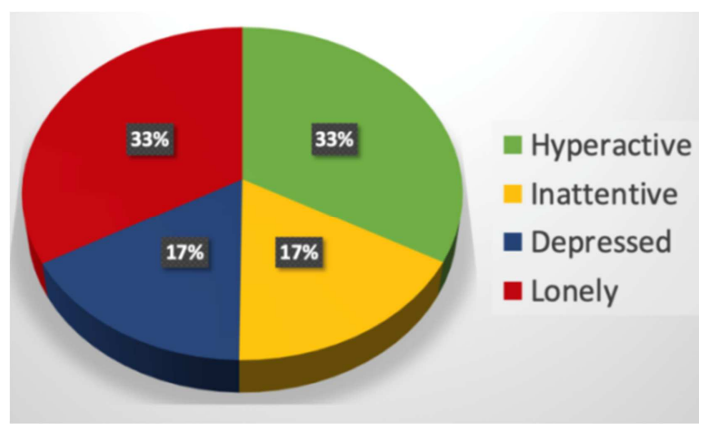

Figure 4. Newly noted symptoms during the lockdown period.

Interestingly, the highest reported new impact is loneliness $(n=238 / 658)$. Figure 5, shows the percentages of the reported new difficulties compared to the total number. Loneliness and hyperactivity represent more than $70 \%$ of reported cases while depression and lack of attentiveness are equally reported and together represent more than $30 \%$.

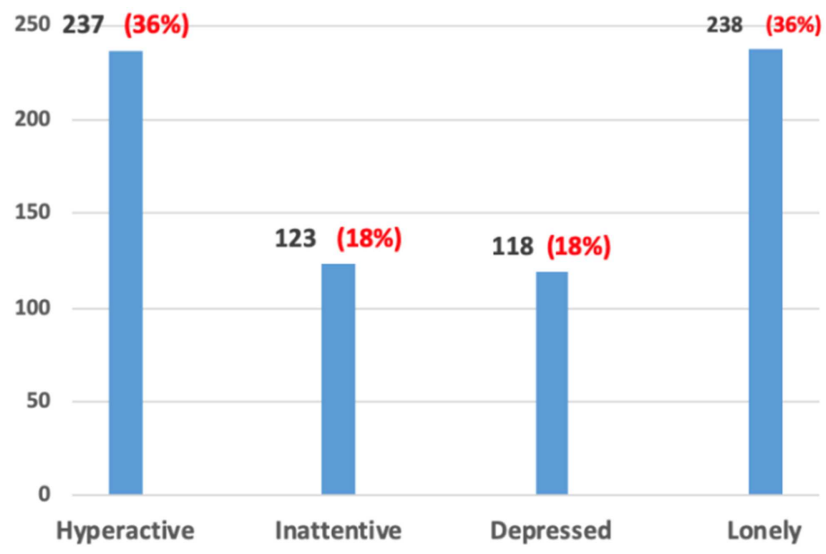

Figure 5. Newly noted symptoms during the lockdown period.

3. Physical Activity and Lack of Space: 
The majority of parents $(72 \% n=471 / 658)$ reported that children physical activities were affected by the lockdown despite only $(50 \% n=330 / 658)$ of the parents reporting lack of space at homes (figure 6). Many parents reported that children were spending a long time on screens not only to do their online school sessions but on games and iPads. Some parents reported increase of weight, laziness and sleep difficulties related to lack of activity. The lack of physical activity is not related to the lack of space at homes only but many of the children were attending out-door various sport activities before the lockdown hence the discrepancy of lack of space and number of affected children in figure 6.
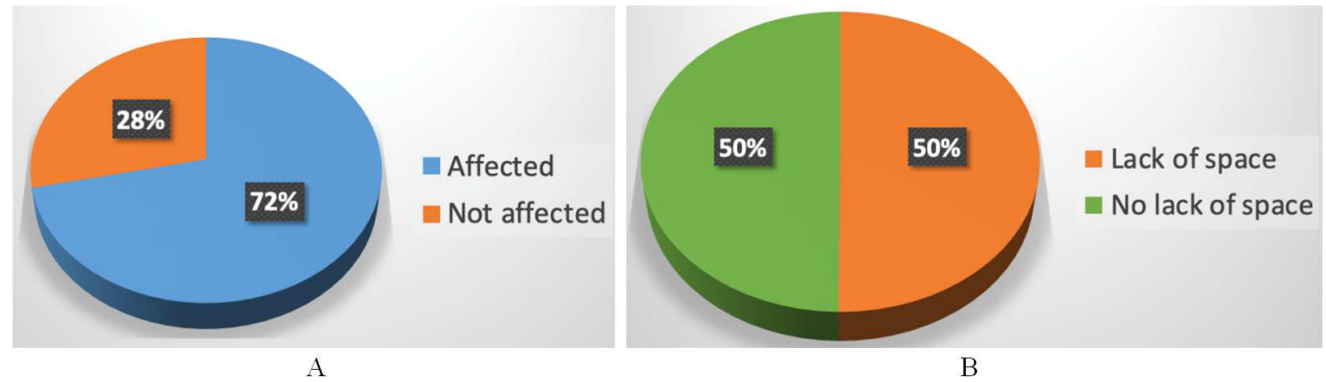

Figure 6. The impact of lockdown on physical activities of children (A) impact on physical activity (B) lack of space.

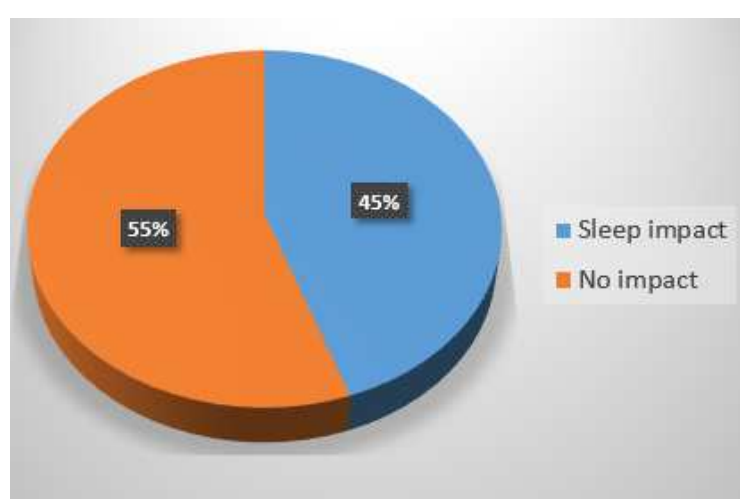

Figure 7. The impact of lockdown on children's' sleep.

\section{Sleep Difficulties:}

Significant number of children $(41 \% n=273 / 658)$ showed new sleep difficulties temporally related to lockdown (figure 7). Most reported difficulties are related to disrupted sleep, sleeping and awaking late, sleeps more, sleep less, having insecurity, speak during sleep, having nightmares, irregular pattern of sleep and worried about parents' jobs. Sleep late and wake late were the most commonly reported difficulties.

5. Eating Problems:

Altered eating habits are one of the most important newly reported impacts because of lockdown. Around half of parents $(48 \% n=314 / 658)$ reported eating difficulties in their children that emerged during lockdown (figure 8). Some of the children who were reported to have eating problems had more than one eating problem. The most commonly reported were junk food, food refusal and picky eating. Junk food eating was the highest reported $(51 \% n=172 / 339$ report $)$ while picky eaters and food refusal nearly equally reported $(26 \% \quad n=87 / 339$ report $)$ and $(23 \% \quad n=80 / 339$ report $)$ respectively. Only a few parents reported a positive effect on eating home cooked food and healthy snacking.
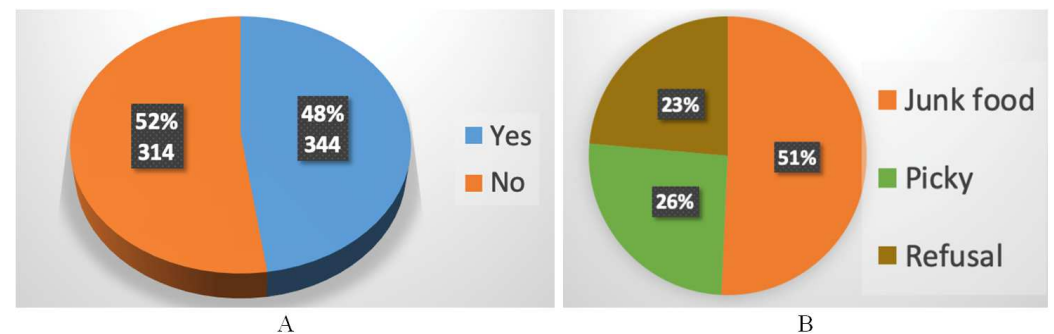

Figure 8. The impact of lockdown on eating (A) number of children have newly reported eating problems (B) the proportion of the commonly reported difficulties.

\section{Psychological Support:}

Despite mental, behavioral, sleep, eating problems and other impacts $(82 \% \mathrm{n}=547 / 658)$ of parents reported no psychological support from professionals such as a psychologist, educational counsellor or others (figure 9). The $83 \%$ may be even more because some parents may answer "yes" as thinking that the mental support means provided by them.

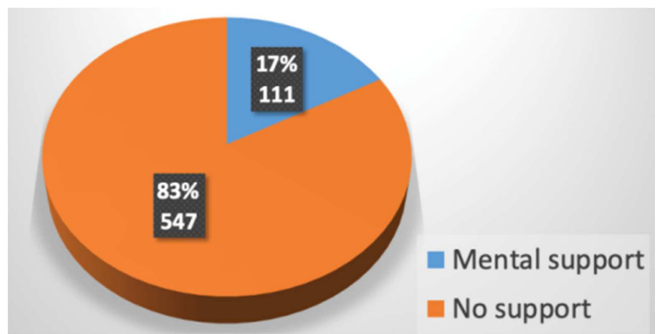

Figure 9. Provision of mental support from professionals or schools. 


\section{Missing Schools and Friends:}

More than $(75 \% \mathrm{n}=494 / 658)$ of children verbalized they were missing school frequently to their parents irrespective of age, gender and culture.

8. COVID-19 Infection in Family and the Impacts:

About $(9 \% \mathrm{n}=58 / 658)$ of surveyed parents reported COVID-19 infection in one or more of immediate family living on the same house (figure 10 and table 4)

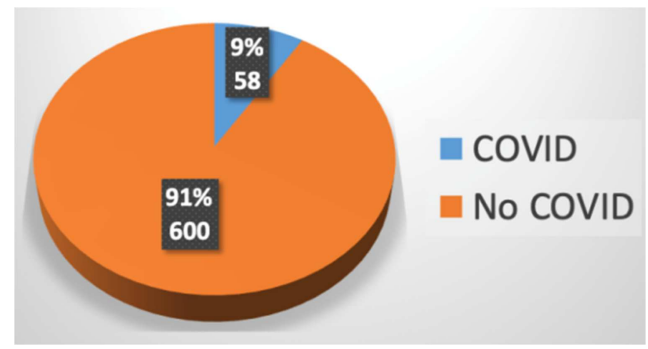

Figure 10. Number of COVID-19 in families.

Table 4. Number of COVID-19 reported in families and nationalities.

\begin{tabular}{lll}
\hline Nationality & Number COVID Cases & Percentage from nationality \\
\hline Algeria & 1 & $* 100$ \\
Filipino & 10 & 14 \\
French & 3 & 30 \\
Indian & 14 & 8 \\
Jordan & 2 & 7 \\
Lebanese & 1 & 12 \\
Malaysia & 1 & 10 \\
Pakistan & 9 & 11 \\
RSA & 2 & 40 \\
Russia & 1 & 30 \\
UAE & 9 & 19 \\
UK & 5 & 11 \\
Total & 58 & 9 \\
\hline
\end{tabular}

*Only one patient.

Figures 11, 12 and 13 show that those who reported COVID-19 infections in one or more household member also reported more impacts on behaviors ( $72 \%$ cf $52 \%)$, sleep $(72 \%$ cf $38 \%$ ), eating (72\% cf $45 \%$ ) and depression (37\% cf 16\%). Mental impacts, parents feeling about long-term impacts beyond lockdown and fear of sending children to public places are more or less same in the two groups (COVID-19 in family compared to no COVID-19 in family).

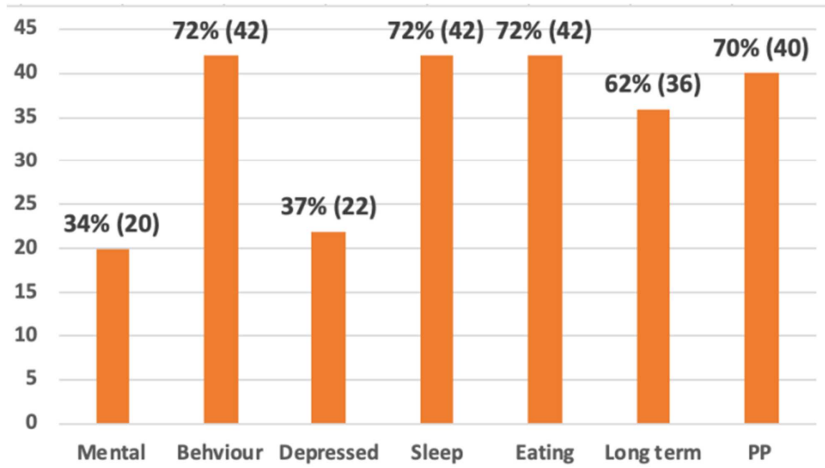

Figure 11. The various impacts of lockdown in COVID-19 infected families (PP=lack of confidence to visit public places after ease of lockdown) (long term see form figure 1 ).

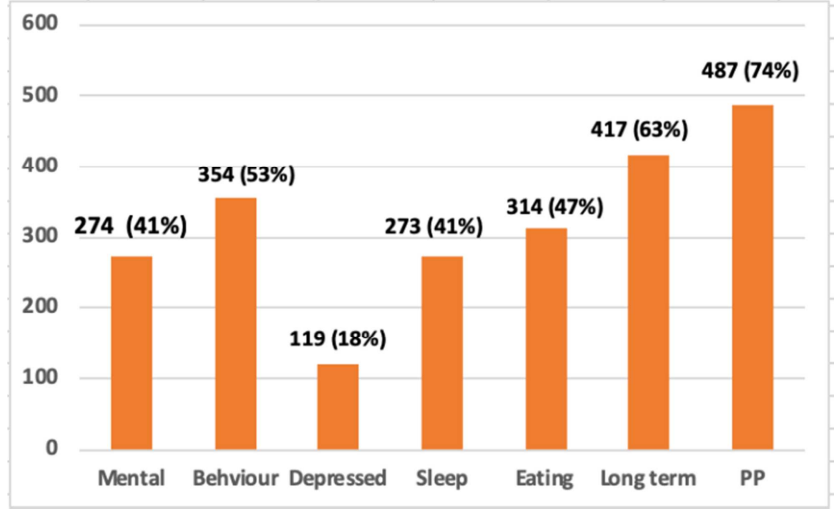

Figure 12. The various impacts of lockdown in all children ( $P P=$ lack of confidence to visit public places after ease of lockdown).

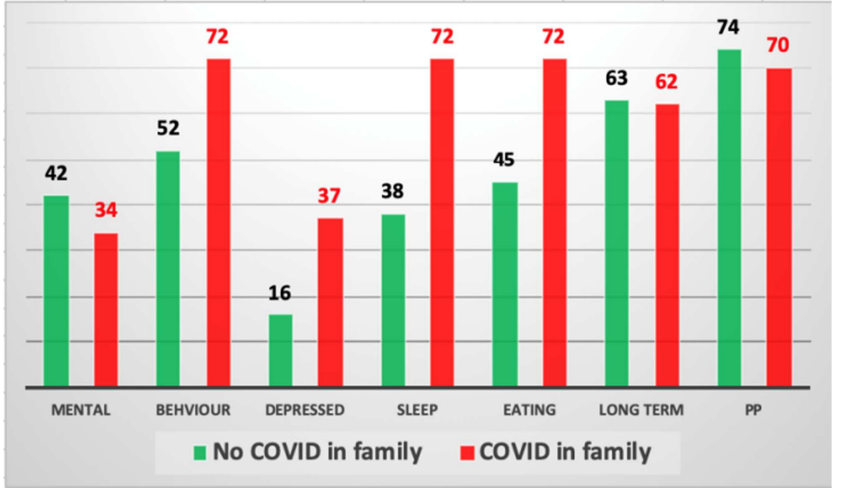

Figure 13. Comparison of the various impacts in (percentages to the total of each) between patients with no COVID in family (600) and those with COVID in family (58).

\section{Vaccinations Difficulties during Lockdown:}

One of the most affected aspects during the lockdown is to bring the children for vaccination in the health centers or hospitals and clinics. We assessed this issue by asking the parents a direct question if the lockdown interfered with vaccinations and development assessment. A third of the parents $(31 \% n=202 / 658)$ answered that fear of infection made them postpone children's vaccinations and or other health assessment. In addition to the fear of infection, other parents reported that the clinics were closed, mother was pregnant, and some lost their insurance. Interestingly when parents were asked if they are confident to visit clinics and health facilities for vaccinations and assessment after lockdown lifted, still one third of them $(32 \% n=212 / 658)$ answered that the fear of infection was still the main cause for their lack of confidence to visit clinics yet.

10. Visiting Public Places with Children:

More than $70 \%(74 \% \mathrm{n}=487 / 658)$ of parents were reluctant to visit public places, shopping centers with their children and or sending children for sports or children centers including schools.

11. Online Virtual Schooling:

During the confinement, schools became virtual, and education provided online from homes. We asked parents about their experience with online teaching and to score such experience between 1 and 5 where 1 is very poor and 5 
excellent. Figures 14 and 15 shows that most parents were satisfied with the level of online schooling as $(65 \%$ $\mathrm{n}=412 / 658)$ scored $3-5$ scoring and only $(35 \% \mathrm{n}=220 / 658)$ scored less than 3. Some parents commented that online schooling produced more work for parents, parents took the role of the teacher, parents are tired, difficult when having more than one child, depending on the teacher, online cannot replace coaching in school, do not know how to teach and difficult to keep the young child concentrated. Quite few parents felt that the whole online learning was introduced in a rush leaving a large burden on parents. A few parents reported that learning at home offered one to one teaching which gave positive outcome.

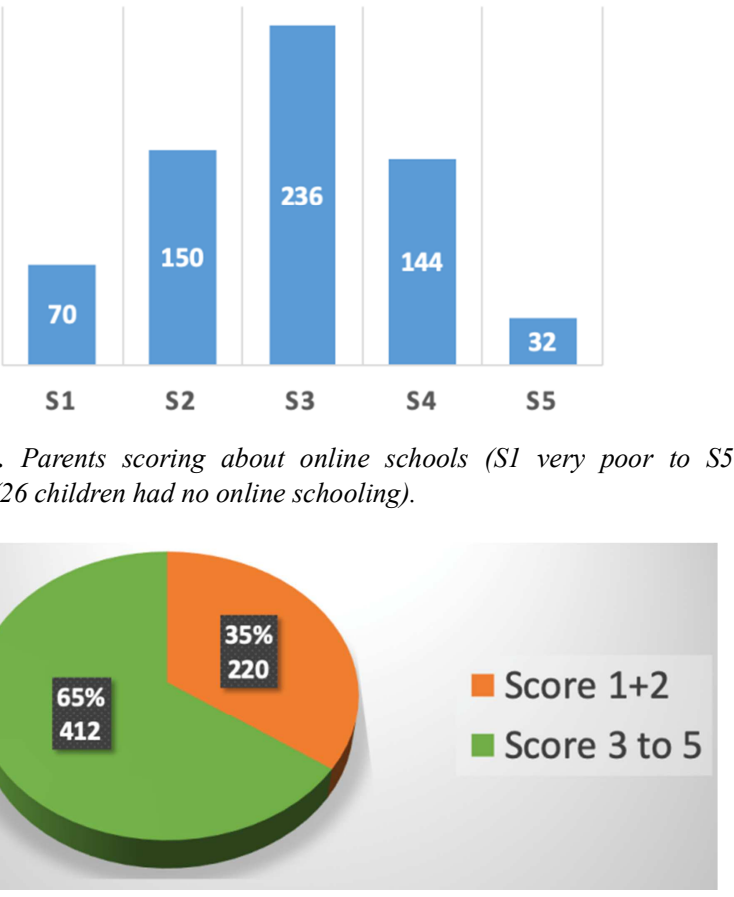

Figure 15. Parents' satisfaction with online schooling.

\section{Long-term Impacts:}

When asked if the home confinement due to COVID-19 may lead to long term impacts such as boredom, fear of infection and or stress $(63 \% n=417 / 658)$ of parents believe it may cause long term impacts that may continue beyond the lockdown period. The distribution of these 3 areas are as shown in figure 16.

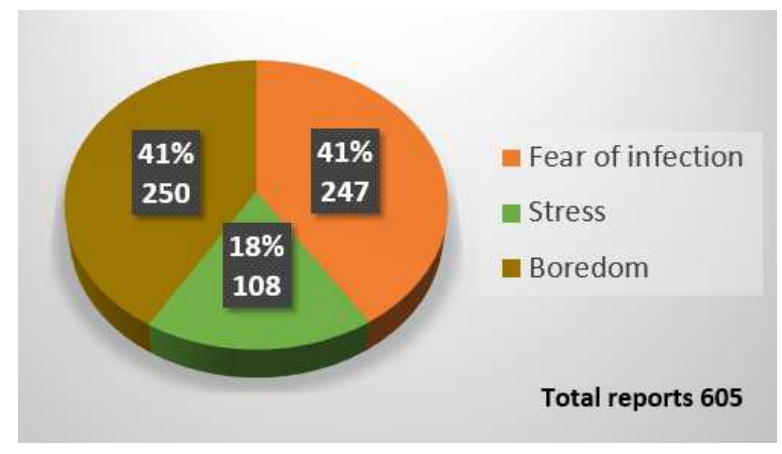

Figure 16. Long-term impacts beyond the lockdown period.
The long-term impacts of fear of infection, stress and boredom are connected to each other and to both the infection and its lockdown. Interestingly, the fear of infection and stress represent the same ratio and likely linked to the pandemic infection while boredom seems to be related to the lockdown itself

\section{Most influencing Source of information for parents:}

The majority of parents $(52 \% n=346 / 658)$ said that the media, including the internet and social media, played a major role in their decision and information taking. Health services and authorities contributed to $(42 \% \mathrm{n}=28 / 658)$ while friends and relative effects contribute to $(37 \% \mathrm{n}=247 / 658)$ only (figure 17).

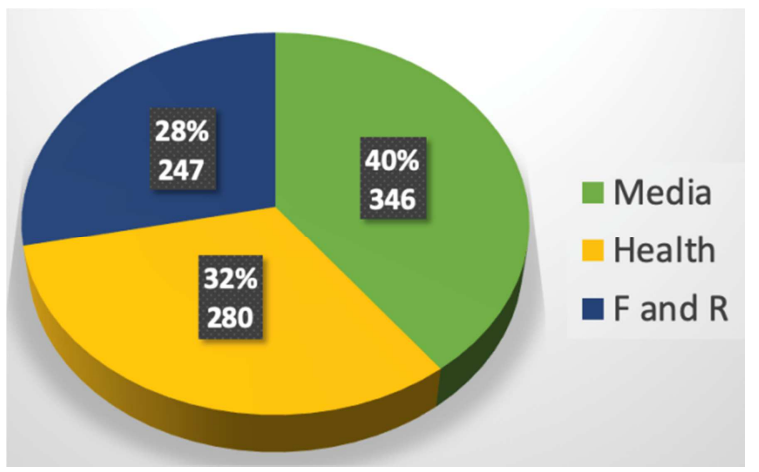

Figure 17. The effects on parents taking information and decision ( $F$ and $R=$ friends and relatives).

Some parents think that media was over-exaggerating, others who answered yes for relatives or friends are in fact health service relatives or friends or doctors, and those who answered yes for health services based their information from the media related health services.

\section{Discussion}

The impacts of home confinement due to COVID-19 resulted in short term and long-term consequences beyond the confinement time. The majority of parents in this study, irrespective of nationalities and social backgrounds reported that their children showed new harmful changes to their behavior, mental, attention, psychological, social, loneness feeling, learning, and physical activities as a result of lockdown. There are significant undesirable impacts on eating and sleep too. Regression in achieved sociodevelopmental skills and newly acquired tasks such as music and sport were reported. More than $65 \%$ of parents believed that these impacts might continue beyond the confinement time such as "fear of infection, boredom and stress" (figure 16). This may be partly related to the effects of the confinement on the hypothalmo-pituitary function that may lead to significant physical and psychological trauma such as slow reaction, lack of attentiveness, symptoms of depression, impulsiveness, stress, psychological trauma and hyperactivity [11]. Children may take longer time to fine-tune to the norm of life again after schools reopening, particularly with newly implemented anti-COIVD-19 preventative measures. In this 
study parents reported that the long-term impacts due to lockdown may be related to many factors such as social isolation, addiction to screen, fear of infection, regression of acquired social skills, fear of trying new things, laziness of adaptation, persistence of lack of physical activity, and getting accustomed to online sessions. Long-term and shortterm impacts due to unprecedented pandemics and disasters were reported in adults but scarcely in children particularly from parent's perspectives of view [12, 13]. On the other hands, psychosocial strains on adults themselves [14] may result in ineffective care of children. In this study mental distress and behavioral difficulties were reported 628 times which is equal to $95 \%$ of the number of children included (figure 3). It highlighted the scale of the impacts of lockdown on children psychological health, which may even manifest later leading to consequences on mental and physical health and productivity at work during adult life [15]. Despite of that there is lack of professional psychological support such as from educational psychologists or school counselor, which reported by $80 \%$ of parents (figure 9 ).

Sleep difficulties that were not existed before the confinement represented a big challenge to parents. There are many factors affecting sleep while in home confinement. In adults, stress, lack of financial security, working from home, lack of physical exercises, social isolation, and worried about future were reported before [16]. In this study around half of parents (figure 7) reported new sleep difficulties after the lockdown. Even parents who achieved better results stated that they worked hard to maintain strict circadian schedule by using different strategies such as games, stories, reward system, fun and adventure out of the lockdown. The younger the children, the more the impacts on sleep. This is more likely due to hypersensitivity of the biological rhythms of sleep and food in young children. Many parents stated that the over use of screens including online schoolings is one of the main causes of sleep problem. The same was observed in other studies [17]. Avoiding the over-use of screens and agreeing a written plan with reward system may help. It will also help in managing conflict resulting from younger children mimicking the older for computers and TV watching. It is likely that sleep difficulties resulted also from other multiple factors such as eating problems, lack of physical activities, stress, and insecurity.

Significant numbers of parents $(75 \% \mathrm{n}=494 / 658)$ (figure 19) reported that their children voiced repetitively that they miss their schools and friends and as a result they felt lonely, bored and sad which may be the first sign of depression. About $15 \%$ of parents felt that their children were showing signs of depression. Unlike online teaching, schools have a critical role in offering an opportunity for students to interact with teachers and obtain psychological and educational counselling when needed.

Most of children in this study were primary or pre-primary age $(85 \% \mathrm{n}=562 / 658)$ (figures 2 and 18). In this age group, sensory play and activities represent a major part of school attendance and is fundamental for brain neuro-connectivity and development. Moreover, younger children are more prone for mental distress which further impair the sensory function and learning [18]. Providing opportunities for children to explore their world through sensory play and learning is crucial. It will mature the child's ability to complete more complex tasks and supports cognitive growth, language development, gross motor skills, social interaction and problem-solving skills. This cannot be achieved by online teaching solely. The ideal way to activate these skills is when the child is with their own peers, teachers and a real world opposite to a virtual world. During home confinement parents may find themselves playing the role of the nursery or school to stimulate the sensory paly which may be provided by sensory books, mouldable sand, sensory bag fillers, bubbles or pouring and transferring activities for food or liquids and music. Sensory play helps the young child to develop auditory processing, oral sensory processing, differentiating and trusting different textures, learning how to achieve calmness, developing healthy eating habits, and establishing cognitive abilities. Sudden interruption of such important activities will result in significant impacts like the ones we reported in this study that need to be addressed with proper home care and parenting.

Although $65 \%$ of parents reported satisfaction with online schooling (figure 15) most of them reported difficulties to achieve this in particular when there is more than one child in the family. There are many difficulties reported with online schooling such as the number of children included, the noises, the tech connectivity, the session's contents, the financial tech implications, and in many instances leaving the parents to deal with teaching.

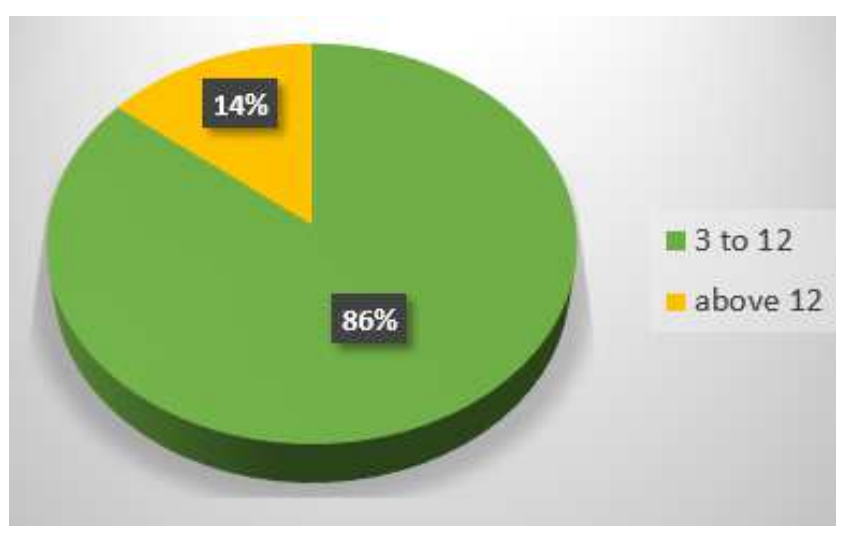

Figure 18. Age of children in the study in years.

Most important reported difficulty by the parents is the unanticipated rapid transition from scheduled teaching to online learning which left teachers and parents unprepared. This leads to wider differences in scores about online teaching due to variation in the individual teachers (figure 9). The online teaching could have been better if the individual variation narrowed by prior training which parents understand due to the lack of time in this unprecedented pandemic. Other important issues as per our parent's comments is the age of the child. The older the child the better the participation and the less supervision and the more ability to use hi tech to communicate. 


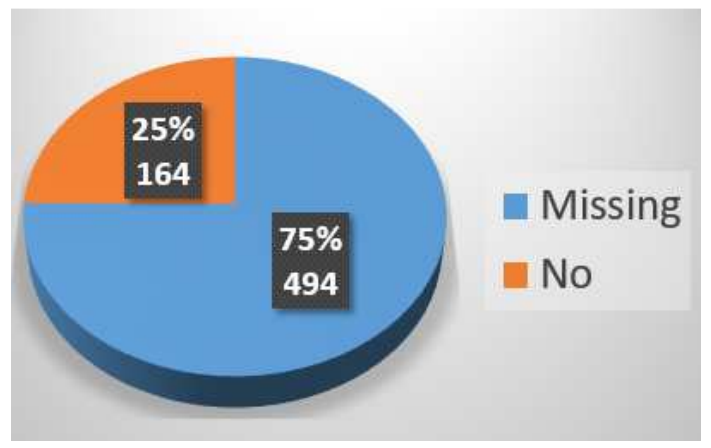

Figure 19. Children verbalized missing school and friends.

All the reported impacts were happening irrespective of whether COVID-19 infection happened in families; however, the percentages of these impacts are more when there is COVID-19 infection happened in the family (figure 13). Behavioral symptoms were more in families with COVID-19 (72\% against $52 \%$ ), depression (37\% against $16 \%$ ), sleep difficulties ( $72 \%$ against $38 \%$ ), eating problems ( $72 \%$ against $45 \%$ ). Interestingly both groups equally feel that the lockdown will lead to long-term consequences, mental difficulties nearly equal as well as the long-term impact and decline to go to public places even after lockdown. More interesting the group with COVID-19 reported double the percentage of depression in children. We may conclude therefore that child who are infected or having infected family members are more prone to the lockdown effects and they more in need of mental health and school support particularly after schools reopen.

It is important to note too that confinement at home may result in weakening of the immune system. Two factors are important; one is the cessation of herd immunity including the corona common viruses that may provide cross cover immunity from SARS-CoV-2 [20]. The second factor is, lack of exposure to sunlight and hence vitamin D deficiency which is an important factor for the immune system and for the mental health as deficiency may cause depression and fatigability [21]. Light-skinned individuals require 20 minutes exposure to direct sunlight 3-4 times a week, whereas darker skin require 30-40 minute exposure 3-4 times a week. It is recommended to expose $18 \%$ of the body, that is arms without long sleeves and face without sunscreen. The ideal exposure time is between 11:00 am and 2:00 pm. Most of the parents we surveyed confirmed that their children lacked these amounts of sun exposure during the lockdown. More studies certainly needed in these aspects too.

In regards to the vaccinations the impact of the lockdown on the vaccinations decreasing is significant and may lead to serious implications. Our results showed that more than $30 \%$ $(31 \% n=202 / 658)$ of parents were not confident and declined to take their children for vaccination mainly due to fear of infection by COVID-19. We noticed from our practice (unpublished data) that the number of missed or postponed vaccinations were significantly higher after COVID-19. According to the WHO and UNICEF data of 15 July 2020
[22] that the COVID-19 confinement and fear of attending for vaccinations or closure of clinics resulted in an alarming decline in the number of children receiving lifesaving vaccines around the world. There is a substantial drop in the number of children completing three doses of the vaccine against diphtheria, tetanus and pertussis (DTP3). This is the first time in 28 years that the world could see a reduction in DTP3, coverage which is the marker for immunization coverage within and across countries. There was serious drop in MMR vaccinations added to the decline noticed over many years and the spread of Measles, whopping cough and mumps across the world. According to the report $75 \%$ of 82 countries reported disruption of their vaccination program due to covid-19. It is therefore very important for the health professionals and maybe schools to monitor children vaccinations.

Consequences of the lockdown after reopening schools need comprehensive action by all professionals involved with children care such as parents' groups, educational psychologists, school counselors, social workers, and clinicians. It is vital to have mechanisms in place for early detection of signs of deterioration of mental health, such as anxiety and depression, sudden changes in behavior, unusual persistent sadness, excessive worry, lack of concentration, trouble sleeping, or exhaustion. Increasing communication with children to address their fears and concerns, playing collaborative games to alleviate loneliness, encouraging activities that promote physical activity, and using music therapy in the form of singing to reduce the worry, fear, and stress that the child may feel should be extended beyond lockdown and after school reopened. Furthermore, parents should pay attention to sleep difficulties and nightmares, prevent increased daytime sleep and suggest sleep hygiene and relaxation methods, model a positive psychological attitude to reduce stress, and divert attention to more productive and positive directions. Moreover, schools should have plans if an outbreak happens and the flexibility to opt to online teaching again if needed temporarily or long time [19].

Schools should be able to screen for symptoms of COVID19. Ideally this should be initiated by parents prior to the child arriving at school. The commonest symptoms of COVID-19 in children are cough, breathing difficulty, fever, headache, earache, poor appetite, lethargy, sore throat, skin rashes, diarrhea, vomiting, enlarged lymph node and insomnia. Schools and parents should have plans of referring symptomatic children for proper medical assessment and an immediate isolation when needed. However, even with strict symptoms, monitoring there is a problem with mildly infected children and those who remained asymptomatic. In a recent study in South Korea [23] 22\% of COVID-19 positive children were completely asymptomatic and 20\% turn symptomatic later on after the swab results. This will certainly represent a dilemma for schools and parents as well as the community because these children remain silent sources for COVID-19 spread. Interestingly adult studies before showed $40 \%$ of COVID-19 positive adults are 
asymptomatic [24].

In schools, children above 2 years can wear masks and or desk screen with ideal distancing (6 feet) [25]. Staff should have access to personal protective equipment (PPE), preventing logjams, frequent cleaning of surfaces, play tools and rest rooms, teachers change class and not students, small classes, adequate aeration, and do not use nebulizer treatment but inhalers with spacer devices. Schools may need to invest in educational psychologists, school nurses and counselors to help to alleviate the mental, behavioral and other impacts shown in our study particularly with the lack of such support as shown too.

Lastly, the flu vaccine in this season is extra-important to protect children above 6 months from acquiring influenza infection which is associated with high morbidity and mortality. It has been shown that $80 \%$ of children die from influenza infection are unvaccinated and it may lead to sever debilitating hemorrhagic encephalitis and death [26, 27]. If COVID-19 and influenza occurred in the same individual or child it will lead to more damage for the respiratory and other systems. Protecting against influenzas is therefore extra important in this season.

\section{Conclusion}

We conclude that the COVID-19 lockdown and fear of infection impacted significantly on children of school ages. It negatively impacted their mental, behavioral, physical, sleep, and eating health. There is a decline in vaccination rate. The impacts may extend beyond the period of home confinement for a long time to come. There is an emergent need to support mental and psychosocial well-being of children and their parents during similar outbreaks to minimize the psychosocial impacts. Mental and psychosocial health initiatives should focus on educating the parents, teacher and health care workers on how to appropriately deal with the enormous pressure and stress. Planning for school reopening with flexibility of opting to virtual teaching again if an outbreak happens in school are important and in need of proactive strategies.

\section{Authors' Contributions}

All the authors contributed to the article. Dr. Sam Hassan conceptualized and designed the study, wrote the article and revised the manuscript and results. Dr. Mary Saviour revised the methodology and statistics. Other authors involved in data collection, analysis, manuscript review and approved the final manuscript as submitted and agreed to be accountable for all the work.

\section{Disclosure Policy}

All authors declare that there is no conflict of interest to disclose regarding the publication of this paper. There are no external funding for this manuscript and all authors have indicated they have no financial relationships relevant to this article to disclose.

\section{Acknowledgements}

Thanks to go to the parents of children who contributed and liked this survey. Acknowledgment to go to Dr Rolf Hartung our Medical Director for his support about the research.

\section{References}

[1] World Health Organization: WHO Coronavirus Disease (COVID-19); Dashboard; https://www.worldometers.info/coronavirus/.

[2] Brazendale K, Beets MW, Weaver RG, et al. Understanding differences between summer vs. school obesogenic behaviors of children: the structured days hypothesis. Int J Behav Nutr Phys Act. 2017; 14: 100.

[3] Sprang G, and Silman M. Posttraumatic stress disorder in parents and youth after health-related disasters. Disaster Med Public Health Prep. 2013; 7 (1): 105-110. doi: 10.1017/dmp.2013.22.

[4] Qiu J, Shen B, Zhao M, et al. A nationwide survey of psychological distress among Chinese people in the COVID19 epidemic: implications and policy recommendations. Gen. Psychiatr 2020; 33, e100213. doi: 10.1136/gpsych-2020100213.

[5] Wang C, Pan R, Wan X, et al. Immediate Psychological Responses and Associated Factors during the Initial Stage of the 2019 Coronavirus Disease (COVID-19) Epidemic among the General Population in China. Int. J. Environ. Res. Public Health 2020; 17. doi: 10.3390/ijerph17051729.

[6] Purssell E, Gould D, Chudleigh J. Impact of isolation on hospitalised patients who are infectious: systematic review with meta-analysis. BMJ open 2020; 10 (2). http://dx.doi.org/10.1136/bmjopen-2019-030371.

[7] Jiao WY, Wang LN, Liu J et al; Behavioral and emotional disorders in children durig COVID-19 epidemic; The J of Ped; 2020; 221: 264-266: 203 doi: https://doi.org/10.1016/j.jpeds.2020.03.013.

[8] Mahase E. Covid-19: Mental health consequences of pandemic need urgent research, paper advises. BMJ 2020; $\begin{array}{lllll}\text { Published } & 16 & \text { April } & 2020 .\end{array}$ https://doi.org/10.1136/bmj.m1515.

[9] American Psychiatric Association; Diagnostic and statistical manual of mental disorders. 5th ed. American Psychiatric Publishing, Arlington (VA) 2013.

[10] Population of Dubai by age group 2018, published 6 March 2020 by Statista Research Department; https://www.statista.com/statistics/724178/dubai-populationage-group/.

[11] Zhang M, Li X, Li J, Sun H, et al. Effects of confinement on physiological and psychological responses and expression of interleukin 6 and brain derived neurotrophic factor mRNA in primiparous and multiparous weaning sows. Asian-Australas J $\begin{array}{llll}\text { Anim Sci. 2017; } 30 & \text { (9): 1350-1357. doi: }\end{array}$ 10.5713/ajas.17.0013. 
[12] Narici M, De Vito G, Franchi M, et al; Impact of sedentarism due to the COVID-19 home confinement on neuromuscular, cardiovascular and metabolic health: Physiological and pathophysiological implications and recommendations for physical and nutritional countermeasures, Europ J of Sport Science, 2020: DOI: 10.1080/17461391.2020.1761076.

[13] Goldmann E and Galea S; Mental Health Consequences of Disasters; 2014; Annual Review of Public Health, 35: 169183. https://doi.org/10.1146/annurev-publhealth-032013182435.

[14] AmmarA, Trabelsi A, Brach $M$ et al; Effects of home confinement on mental health and lifestyle behaviours during the COVID-19 outbreak: Insight from the "ECLB-COVID19" multi countries survey, medRxiv, 2020; doi: https://doi.org/10.1101/2020.05.04.20091017.

[15] Clark H, Coll-Seck AM, Banerjee A et al. A future for the world's children? A WHO-UNICEF-Lancet Commission. Lancet. 2020; 395: 605-658.

[16] Altena E, Baglioni C, Espie CA, et al. Dealing with sleep problems during home confinement due to the COVID-19 outbreak: Practical recommendations from a task force of the European CBT-I Academy. J Sleep Res. 2020; 00: e13052. https://doi.org/10.1111/jsr.13052

[17] Guo J, Chen L, Wang X, et al. The relationship between Internet addiction and depression among migrant children and left-behind children in China. Cyberpsychol Behav Soc Netw. 2012; 15 (11): 585-590. doi: 10.1089/cyber.2012.0261.

[18] Pierce M, Hope H, Ford T et al; Mental health before and during the COVID-19 pandemic: a longitudinal probability sample survey of the UK population; Lancet Psychiatry 2020; Published Online, July 21, 2020, https://doi.org/10.1016/S2215-0366(20)30308-4.

[19] Return to school during COVID-19: https://www.healthychildren.org/English/health-
issues/conditions/COVID-19/Pages/Return-to-School-DuringCOVID-19.aspx.

[20] Sam Hassan. "Presentation, Management and Pathogenesis of the SARS-CoV-2 in Children". Acta Scientific Paediatrics 3.7 (2020): 13-31.

[21] Sam Hassan, Farsheed Zakir, Sameer Sajwani, PostStreptococcal Syndrome in a 5-Years Old Boy with Sever Vitamin D Deficiency Only Responding to Monoclonal Antibody: First Case Report, American Journal of Pediatrics. Vol. 4, No. 2, 2018, pp. 25-30. doi: 10.11648/j.ajp.20180402.12.

[22] https://data.unicef.org/resources/immunization-coverageestimates-data-visualization/.

[23] DeBiasi RL, Delaney M. Symptomatic and Asymptomatic Viral Shedding in Pediatric Patients Infected With Severe Acute Respiratory Syndrome Coronavirus 2 (SARS-CoV-2): Under the Surface. JAMA Pediatr. Published online August 28, 2020. doi: 10.1001/jamapediatrics.2020.3996.

[24] Gao Z, Xu Y, Sun C, et al. A systematic review of asymptomatic infections with COVID-19. J Microbiol Immunol Infect. Published online May 15, 2020. doi: 10.1016/j.jmii.2020.05.001.

[25] Wang CJ and Blair $\mathrm{H}$; Operational considerations on the American Academy of Pedaitrics Guidance for K-12 School Reentry; JAMA Pediatrpublished online Agust 11, 2020. Doi: 10.1001/jamapediatrics.2020.3871.

[26] Flannery B, Reynolds SB, Blanton L, et al. Influenza Vaccine Effectiveness Against Pediatric Deaths: 2010-2014. Pediatrics. 2017; 139 (5): e20164244. doi: 10.1542/peds.2016-4244.

[27] Mastrolia, M. V., Rubino, C., Resti, M. et al. Characteristics and outcome of influenza-associated encephalopathy/encephalitis among children in a tertiary pediatric hospital in Italy, 2017-2019. BMC Infect Dis 19, 1012 (2019). https://doi.org/10.1186/s12879-019-4636-5. 